PROCEEDINGS OF THE

AMERICAN MATHEMATICAL SOCIETY

Volume 126, Number 9, September 1998, Pages 2535-2539

S 0002-9939(98)04320-2

\title{
DIFFERENTIAL FORMS ON QUOTIENTS BY REDUCTIVE GROUP ACTIONS
}

\author{
MICHEL BRION
}

(Communicated by Roe Goodman)

\begin{abstract}
Let $X$ be a smooth affine algebraic variety where a reductive algebraic group $G$ acts with a smooth quotient space $Y=X / / G$. We show that the algebraic differential forms on $X$ which are pull-backs of forms on $Y$ are exactly the $G$-invariant horizontal differential forms on $X$.
\end{abstract}

\section{INTRODUCTION}

Let $G$ be a reductive algebraic group of automorphisms of an affine algebraic variety $X$ over an algebraically closed field $k$ of characteristic zero. Denote by $k[X]$ the algebra of regular functions on $X$. The subalgebra $k[X]^{G}$ of $G$-invariants in $k[X]$ is finitely generated; let $Y=X / / G$ be the affine algebraic variety such that $k[Y]=k[X]^{G}$ and let $\pi: X \rightarrow Y$ be the quotient map defined by the inclusion $k[Y] \subset k[X]$. Then we have a pull-back map $\pi^{*}: \Omega^{*}(Y) \rightarrow \Omega^{*}(X)$, where $\Omega^{*}$ denotes the algebra of Kähler differential forms, see e.g. [K].

In the present note, we describe the image of $\pi^{*}$ in the case where $X$ and $Y$ are smooth. In other words, we characterize the differential forms on $X$ which can be written as sums of products of invariant functions and of their differentials. Clearly, such forms are $G$-invariant and horizontal, that is, their interior product with any vector field induced by the $G$-action is zero. Let $\Omega_{\mathrm{hor}}^{*}(X)^{G}$ be the space of $G$-invariant horizontal forms; such forms are also called basic.

Is any basic differential form in the image of $\pi^{*}$ ? This question was raised by P. Michor in the setting of a representation of a compact Lie group on a real vector space $V$, and of differential forms on $V$ with smooth coefficients (which do not coincide with Kähler differentials for the ring of smooth functions on $V$; but differential forms with polynomial coefficients coincide with Kähler differentials for the ring of polynomial functions). Michor showed that the answer is negative in general, but positive for polar representations; see [M1] and [M2].

Here we obtain the following

Theorem 1. Let $G$ be a reductive group of automorphisms of a smooth, affine variety $X$, and let $\pi: X \rightarrow Y$ be the quotient map. If $Y$ is smooth, then $\pi^{*}$ : $\Omega^{*}(Y) \rightarrow \Omega_{\mathrm{hor}}^{*}(X)^{G}$ is an isomorphism.

This proves a conjecture of V. L. Popov formulated in his talk at the problem session of the conference "Algebraic Groups", Kazimierz (Poland), May 25-June 1,

Received by the editors October 25, 1996 and, in revised form, January 29, 1997.

1991 Mathematics Subject Classification. Primary 14L30, 22E99.

(C)1998 American Mathematical Society 
1996. Some applications, and a generalization to the case of a singular quotient space, are given in $\S 3$ below. The author thanks V. L. Popov, G. Schwarz (who gave another proof of Theorem 1, in the case of a representation of a connected group) and D. Zaitsev (who gave another proof of Theorem 1, in the case where $G$ is finite) for useful discussions. Thanks are also due to the referee for suggesting simplifications of several points in the proof.

\section{Proof of Theorem 1}

Our approach is similar to the one in $[\mathrm{B}], \S 2$. We begin with an observation concerning reflexive modules; this notion of commutative algebra can be defined as follows. Let $A$ be a Noetherian domain, and let $M$ be a finite $A$-module. Denote by $M^{\vee}=\operatorname{Hom}_{A}(M, A)$ the dual module. Then we have a natural map from $M$ to its double dual $M^{\vee \vee}$, and $M$ is called reflexive if this map is an isomorphism.

Lemma 1. Let $A$ be a normal Noetherian ring, let $M, N$ be finite A-modules with $M$ reflexive and $N$ torsion-free, and let $u: M \rightarrow N$ be an A-linear map. If $u$ is an isomorphism in codimension one, then $u$ is an isomorphism.

Proof. Set $Z=\operatorname{Spec}(A)$ and let $U \subset Z$ be the open subset where $u$ is an isomorphism. Denote by $\mathcal{M}, \mathcal{N}$ the sheaves on $Z$ associated to $M, N$. Then we have a commutative square

$$
\begin{array}{ccc}
M & \rightarrow & \Gamma(U, \mathcal{M}) \\
\downarrow & & \downarrow \\
N & \rightarrow & \Gamma(U, \mathcal{N})
\end{array}
$$

By assumption, the map $\Gamma(U, \mathcal{M}) \rightarrow \Gamma(U, \mathcal{N})$ is an isomorphism. Moreover, the codimension of $Z \backslash U$ in $Z$ is at least two, and hence the map $M \rightarrow \Gamma(U, \mathcal{M})$ is an isomorphism; see $[\mathrm{H}]$, Proposition 1.6. Finally, the map $N \rightarrow \Gamma(U, \mathcal{N})$ is injective because $N$ is torsion-free. These three facts imply our statement.

We will check that Lemma 1 applies to the ring $k[Y]$ and to the map $\pi^{*}$ : $\Omega^{*}(Y) \rightarrow \Omega_{\mathrm{hor}}^{*}(X)^{G}$. Observe that both $\Omega^{*}(Y)$ and $\Omega_{\mathrm{hor}}^{*}(X)^{G}$ are $k[Y]$-modules, and that $\pi^{*}$ is $k[Y]$-linear. Because $Y$ is smooth, $\Omega^{*}(Y)$ is locally free over $k[Y]$, and hence reflexive. Moreover, the $k[X]$-module $\Omega^{*}(X)$ is finitely generated and torsion-free. Therefore, the same holds for the $k[Y]$-module $\Omega^{*}(X)^{G}$ (see [P-V], Theorem 3.24) and for its submodule $\Omega_{\mathrm{hor}}^{*}(X)^{G}$.

So it suffices to check that $\pi^{*}$ is an isomorphism in codimension one. For this, we will use Luna's slice theorem, see [L1]. First recall a

Definition. An equivariant morphism $f: X^{\prime} \rightarrow X$ of affine $G$-varieties is excellent if

(i) $f$ is étale,

(ii) the induced morphism $f / / G: X^{\prime} / / G:=Y^{\prime} \rightarrow Y$ is étale, and

(iii) the morphism $(f, \pi): X^{\prime} \rightarrow X \times_{Y} Y^{\prime}$ is an isomorphism.

Let $y \in Y$. The fiber $\pi^{-1}(y)$ contains a unique closed orbit, say $G \cdot x$, and the isotropy group $G_{x}:=H$ is reductive. By Luna's slice theorem, we have a locally closed smooth, affine, $H$-stable subvariety $S \subset X$ which contains $x$, with the following properties:

(1) $N:=T_{x} S$ is an $H$-complement to $T_{x}(G \cdot x)$ in $T_{x} X$.

(2) The canonical map $G \times{ }^{H} S \rightarrow X$ is excellent. 
(3) There is an excellent $H$-morphism $S \rightarrow N$ sending $x$ to the origin in $N$. (Hence the induced map $G \times{ }^{H} S \rightarrow G \times{ }^{H} N$ is excellent.)

We will also need the following

Lemma 2. Let $f: X^{\prime} \rightarrow X$ be excellent, and set $Y^{\prime}:=X^{\prime} / / G$. Then both maps $k\left[Y^{\prime}\right] \otimes_{k[Y]} \Omega_{\mathrm{hor}}^{*}(X)^{G} \rightarrow \Omega_{\mathrm{hor}}^{*}\left(X^{\prime}\right)^{G}$ and $k\left[Y^{\prime}\right] \otimes_{k[Y]} \Omega^{*}(Y) \rightarrow \Omega^{*}\left(Y^{\prime}\right)$ are isomorphisms of $k\left[Y^{\prime}\right]$-modules.

Proof. Because $f / / G$ is etale, the natural map $u: k\left[Y^{\prime}\right] \otimes_{k[Y]} \Omega^{1}(Y) \rightarrow \Omega^{1}\left(Y^{\prime}\right)$ is surjective (indeed, the cokernel of $u$ is the space of relative differentials $\Omega^{1}\left(Y^{\prime} / Y\right)$ which vanishes because $f / / G$ is unramified). Moreover, because $Y$ is smooth and $f / / G$ is etale, $Y^{\prime}$ is smooth, too. Therefore, the $k\left[Y^{\prime}\right]$-modules $k\left[Y^{\prime}\right] \otimes_{k[Y]} \Omega^{1}(Y)$ and $\Omega^{1}\left(Y^{\prime}\right)$ are locally free of $\operatorname{rank} \operatorname{dim}\left(Y^{\prime}\right)=\operatorname{dim}(Y)$. It follows that $u$ is an isomorphism. This implies the second statement.

Because $k\left[X^{\prime}\right]=k[X] \otimes_{k[Y]} k\left[Y^{\prime}\right]$ and $f$ is etale, there are equivariant isomorphisms $k\left[Y^{\prime}\right] \otimes_{k[Y]} \Omega^{*}(X) \rightarrow k\left[X^{\prime}\right] \otimes_{k[X]} \Omega^{*}(X) \rightarrow \Omega^{*}\left(X^{\prime}\right)$. This implies the first statement.

Using the above and Lemma 2 twice, one reduces Theorem 1 to spaces of the form $G \times{ }^{H} N$. Now, by Lemma 4.1 in [M1], restriction to $N \subset G \times{ }^{H} N$ induces an isomorphism $\Omega_{\mathrm{hor}}^{*}\left(G \times{ }^{H} N\right)^{G} \simeq \Omega_{\mathrm{hor}}^{*}(N)^{H}$, so we can reduce to the case of representations. Moreover, writing $N=N^{H} \oplus V$ as an $H$-representation, we can reduce to considering the action of $H$ on $V$, since the extra trivial factor just tensors the algebras $k[V]$ and $k[V]^{H}$ with $k\left[N^{H}\right]$ and the spaces $\Omega_{\mathrm{hor}}^{*}(V)^{H}$ and $\Omega^{*}(V / / H)$ with $\Omega^{*}\left(N^{H}\right)$.

Moreover, by Lemma 1, we may replace the quotient $Y$ by any open subset $U$ such that $Y \backslash U$ has codimension at least two in $Y$. Recall that $Y$ is stratified by slice types $(H, N)$; see [L1]. If $y \in Y$ is in the open stratum (resp. in a stratum of codimension one), then $V / / H$ is a point (resp. is one-dimensional). So we can finally reduce to the case of a reductive group $H \subset \mathrm{GL}(V)$, where $V^{H}=0$ and $\operatorname{dim}(V / / H) \leq 1$. Then the proof is concluded by the following lemmas.

Lemma 3. In the case where $V / / H$ is a point, the spaces $\Omega_{\mathrm{hor}}^{p}(V)^{H}$ vanish for all $p \geq 1$.

Proof. We may assume that $H$ is connected. First consider the case where $H$ is a torus. Then, because $V / / H$ is a point, all weights of $H$ in $V$ lie in an open half-space. So the weights of $\wedge^{p} V^{*}$ lie in the opposite half-space. Observing that $\Omega^{p}(V) \simeq S^{\bullet}\left(V^{*}\right) \otimes \wedge^{p} V^{*}$, where $S^{\bullet}\left(V^{*}\right)$ is the symmetric algebra of $V^{*}$, we obtain $\Omega^{p}(V)^{H}=0$ for $p \geq 1$, which implies our statement.

In the general case of reductive $H$, let $T \subset H$ be a maximal torus. By the Hilbert-Mumford criterion, there exists a $T$-submodule $W \subset V$ such that $V=H W$ and that all weights of $T$ in $W$ lie in an open half-space. Because the map

$$
\begin{aligned}
f: H \times W & \rightarrow V \\
(g, w) & \mapsto g w
\end{aligned}
$$

is surjective, we can find $w \in W$ such that the differential

$$
\begin{array}{ccc}
d f_{e, w}: & \operatorname{Lie}(H) \times W & \rightarrow \\
(\xi, z) & \rightarrow \xi w+z
\end{array}
$$

is surjective, where $\operatorname{Lie}(H)$ denotes the Lie algebra of $H$. In other words, we have $V=\operatorname{Lie}(H) w+W$. It follows that $\wedge^{p} V=\sum_{q=0}^{p}\left(\wedge^{p-q} \operatorname{Lie}(H) w\right) \wedge\left(\wedge^{q} W\right)$. Let $\omega \in$ 
$\Omega_{\text {hor }}^{p}(V)^{H}$. Because $\omega$ is horizontal, it vanishes on $\sum_{q=0}^{p-1}\left(\wedge^{p-q} \operatorname{Lie}(H) w\right) \wedge\left(\wedge^{q} W\right)$. Moreover, restriction of $\omega \in S^{\bullet}\left(V^{*}\right) \otimes \wedge^{p} V^{*}$ to $W$ is a $T$-equivariant morphism from $W$ to $\wedge^{p} V^{*}$. By the first step of the proof, this morphism vanishes on the subspace $\wedge^{p} W$ of $\wedge^{p} V$. So restriction of $\omega$ to $W$ is trivial. Because $V=H W$ and $\omega$ is $H$-invariant, we conclude that $\omega=0$.

Lemma 4. In the case where $V / / H$ is one-dimensional, the map $\Omega^{1}(V / / H) \rightarrow$ $\Omega_{\mathrm{hor}}^{1}(V)^{H}$ is an isomorphism, and moreover $\Omega_{\mathrm{hor}}^{p}(V)^{H}=0$ for all $p \geq 2$.

Proof. The algebra $k[V]^{H}$ is generated by a non-constant homogeneous function $f$. We have $\Omega^{1}(V / / H)=k[f] d f$ and $\Omega^{p}(V / / H)=0$ for $p \geq 2$. By Lemma 3 and our reductions, the $k[f]$-linear map $\Omega^{*}(V / / H) \rightarrow \Omega_{\mathrm{hor}}^{*}(V)^{H}$ is an isomorphism over the field $k(f)$. Because the $k[f]$-module $\Omega_{\text {hor }}^{*}(V)^{H}$ is torsion-free, it follows that $\Omega_{\text {hor }}^{p}(V)^{H}=0$ for $p \geq 2$ and that $\Omega_{\text {hor }}^{1}(V)^{H}=k[f] \omega$ for some homogeneous form $\omega$. Let $m$ be the degree of $f$, and let $n$ be the degree of $\omega$. Because $d f$ is in $k[f] \omega$, we have $m-1=r m+n$ for some integer $r \geq 0$. This forces $r=0$ and then $\omega$ is a scalar multiple of $d f$.

\section{Some Applications AND A Generalization}

Here are two applications of Theorem 1 which were pointed out by V. L. Popov. First, we recover the following classical result of L. Solomon [So].

Corollary 1. Let $G \subset \mathrm{GL}(V)$ be a finite group generated by pseudo-reflections. Then any $G$-invariant differential form on $V$ is the pull-back of a differential form on $V / G$.

Indeed, the quotient space $V / G$ is smooth, and Theorem 1 applies.

Another application is an algebraic version of a recent result of P. Michor: let $G$ be a Lie group of isometries of a smooth Riemannian manifold $M$. Assume that the $G$-action on $M$ admits a section $\Sigma$, and denote by $W(\Sigma)=N_{G}(\Sigma) / Z_{G}(\Sigma)$ the corresponding "generalized Weyl group". Then restriction of differential forms with smooth coefficients induces an isomorphism $\Omega_{\mathrm{hor}}^{*}(M)^{G} \rightarrow \Omega^{*}(\Sigma)^{W(\Sigma)}$; see [M1], Theorem 3.7, and [M2], Theorem 2.

Corollary 2. Let $X$ be a smooth irreducible affine variety with an action of a reductive group $G$. Denote by $H \subset G$ a principal isotropy group of $X$, by $W$ the quotient group $N_{G}(H) / H$, and by $\Sigma \subset X^{H}$ the closure of the subset of principal points. If the quotient $X / / G$ is smooth, then restriction to $\Sigma$ induces an isomorphism $\Omega_{\text {hor }}^{*}(X)^{G} \simeq \Omega_{\text {hor }}^{*}(\Sigma)^{W}$.

Indeed, we have a commutative diagram

$$
\begin{array}{ccc}
\Omega^{*}(X / / G) & \rightarrow & \Omega^{*}(\Sigma / / W) \\
\downarrow & & \downarrow \\
\Omega_{\text {hor }}^{*}(X)^{G} & \rightarrow & \Omega_{\text {hor }}^{*}(\Sigma)^{W}
\end{array}
$$

where the top horizontal map is pull-back by the morphism $\Sigma / / W \rightarrow X / / G$. But the latter is an isomorphism by the Luna-Richardson restriction theorem; see [L2], Corollaire 4, and [L-R]. So, by Theorem 1, both vertical arrows are isomorphisms.

Finally, Theorem 1 can be extended to the case where the quotient space $Y$ may be singular. Then we have to replace the $k[Y]$-module of Kähler differentials $\Omega^{*}(Y)$ by its double dual $\Omega^{*}(Y)^{\vee}$, the module of Zariski-Lipman differential forms. By adapting the previous argument, we obtain the following 
Theorem 2. Let $G$ be a reductive group acting on a smooth affine variety $X$, and let $\pi: X \rightarrow Y$ be the quotient map. If no divisor in $X$ is mapped by $\pi$ to a subvariety of codimension at least two in $Y$, then $\pi^{*}: \Omega^{*}(Y) \rightarrow \Omega_{\mathrm{hor}}^{*}(X)^{G}$ factors through an isomorphism $\Omega^{*}(Y)^{\vee \vee} \rightarrow \Omega_{\mathrm{hor}}^{*}(X)^{G}$.

In particular, the Zariski-Lipman differential forms on a quotient of a smooth variety $X$ by a finite group are exactly the invariant differential forms on $X$.

Proof. The assumption that no divisor in $X$ is contracted by $\pi$ implies that the $k[Y]$-module $\Omega_{\mathrm{hor}}^{*}(X)^{G}$ is reflexive, using $[\mathrm{H}]$ Proposition 1.6. Therefore, $\pi^{*}$ factors through a map $\Omega^{*}(Y)^{\vee \vee} \rightarrow \Omega_{\text {hor }}^{*}(X)^{G}$. Furthermore, this map is an isomorphism, by Lemma 1, smoothness in codimension one of $Y$, and Theorem 1.

We ignore whether the assumption that $\pi$ contracts no divisor is necessary for Theorem 2 to hold. Observe that the Zariski-Lipman differentials cannot be replaced by Kähler differentials in this statement, as shown by the following example.

Let $G$ be a cyclic group of order $n$ acting on $X=k^{2}$ by scalar multiplication by $n$-th roots of unity. Then the algebra $k[X]^{G}$ is generated by all monomials of total degree $n$ in the coordinates $x_{1}, x_{2}$. Therefore, the differentials of these monomials are the minimal system of homogeneous generators of the $k[Y]$-module $\Omega^{1}(Y)$; this system consists of $n+1$ elements. But the $k[Y]$-module $\Omega_{\mathrm{hor}}^{1}(X)^{G}=\Omega^{1}(X)^{G}$ is minimally generated by $2 n$ homogeneous elements, products of monomials of degree $n-1$ by $d x_{1}, d x_{2}$. So the quotient $\Omega^{1}(X)^{G} / \pi^{*} \Omega^{1}(Y)$ has dimension $n-1$.

\section{REFERENCES}

[B] M. Brion: Sur les modules de covariants, Ann. scient. Éc. Norm. Sup. 26 (1993), p. 1-21. MR 95c: 14062

[H] R. Hartshorne: Stable reflexive sheaves, Math. Ann. 254 (1980), p. 121-176. MR 82b:14011

[K] E. Kunz: Kähler Differentials, Viehweg, Braunschweig-Wiesbaden, 1986. MR 88e:14025

[L1] D. Luna: Slices étales, Bull. Soc. math. France, Mémoire 33 (1973), p. 81-105. MR 49:7269

[L2] D. Luna: Adhérences d'orbites et invariants, Invent. Math. 29 (1975), 231-238. MR $\mathbf{5 1 : 1 2 8 7 9}$

[L-R] D. Luna and R. W. Richardson: A generalization of the Chevalley restriction theorem, Duke Math. J. 46 (1979), p. 487-496. MR 80k:14049

[M1] Peter W. Michor: Basic differential forms for actions of Lie groups, Proc. Amer. Math. Soc. 124 (1996), p. 1633-1642. MR 96g:57041

[M2] Peter W. Michor: Basic differential forms for actions of Lie groups II, Proc. Amer. Math. Soc. 125 (1997), 2175-2177. CMP 97:10

[P-V] V. L. Popov and E. B. Vinberg: Invariant Theory, Encyclopaedia of Mathematical Sciences, Algebraic Geometry IV, vol. 55, Springer-Verlag, Berlin 1994, p. 123-284. MR 92d:14010

[So] L. Solomon: Invariants of finite reflection groups, Nagoya Math. J. 22 (1963), p. 57-64. MR 27:4872

Institut Fourier, B. P. 74, 38402 Saint-Martin D'Hères Cedex, France

E-mail address: mbrion@fourier.ujf-grenoble.fr 